\title{
$\mathrm{C} \mathrm{I} \cdot \mathbf{R}^{\circ} \mathbf{P} \cdot \mathrm{E}^{\mathrm{E}} \mathrm{E}$
}

Centre Interuniversitaire sur le Risque, les Politiques Économiques et l'Emploi

Cahier de recherche/Working Paper 11-32

\section{On Risk Aversion, Classical Demand Theory, and KM Preferences}

Leonard J. Mirman

Marc Santugini

Première version/First Version : Octobre/October 2011

Version révisée/Revised : Décembre/December 2013

Mirman: Department of Economics, University of Virginia, USA

lm8h@virginia.edu

Santugini: Institute of Applied Economics and CIRPÉE, HEC Montréal, Canada

marc.santugini@hec.ca

We are grateful to an Editor and an anonymous referee for their very helpful comments. We thank Elena Antoniadou for arousing our interest in the subject again and Toshihiko Mukoyama for helpful comments. A previous version of this paper entitled "Risk Aversion and Classical Demand Theory" was written with Hou Fei who has withdrawn his name from the project. We thank him for his contributions to the structure of the paper. 


\begin{abstract}
:
Building on Kihlstrom and Mirman (1974)'s formulation of risk aversion in the case of multidimensional utility functions, we study the effect of risk aversion on optimal behavior in a general consumer's maximization problem under uncertainty. We completely characterize the relationship between changes in risk aversion and classical demand theory. We show that the effect of risk aversion on optimal behavior depends on the income and substitution effects. Moreover, the effect of risk aversion is determined not by the riskiness of the risky good, but rather the riskiness of the utility gamble associated with each decision.
\end{abstract}

Keywords: Classical Demand Theory, Consumer Choice, Income and Substitution Effects, Risk Aversion

JEL Classification: D01, D81, D91 


\section{Introduction}

One of the central questions in the field of economics of uncertainty is the influence of attitudes toward risk (i.e., the effect of risk aversion) on optimal decisions. Arrow (1965) and Pratt (1964) first looked at this question in the early 1960s, in the context of a portfolio problem. Since then, the analysis of the behavior of risk-averse individuals facing risk has been set in the context of Arrow-Pratt theory, most notably in the fields of insurance and finance. The analysis has also been extended by Kihlstrom and Mirman (1974) (henceforth, KM) to multidimensional utility functions in situations in which the goods are not perfect substitutes (e.g., a dynamic environment). In particular, KM shows that to generalize the Arrow-Pratt approach to the multidimensional case, the issue of separating tastes from attitudes toward risk must be dealt with. Specifically, the effect of risk aversion on behavior in the multidimensional case must take account of the problem of disentangling tastes and attitudes toward risk. To achieve this, KM considers utility functions that differ by a concave transformation, and, thus, preserve ordinal preferences over gambles.

In this paper, we study the effect of risk aversion on optimal decisions using the KM approach. ${ }^{1}$ Specifically, we consider a general consumer's maximization problem under uncertainty subject to a budget constraint. In the stochastic environment, there is a sure good and a risky good. The good is risky due to the presence of randomness in the budget constraint. The sure good is chosen before the realization of the random variable is observed. The risky good is a residual, i.e., the risky good depends on the outcome of the random parameter through the budget constraint. The set up is thus a generalization of Arrow-Pratt's portfolio model in which the goods are perfect substitutes. We consider three cases of randomness: random income, random price of the sure good, and random price of the risky good. ${ }^{2}$ In each case, we study the effect of risk aversion on optimal decisions.

\footnotetext{
${ }^{1}$ See Diamond and Stiglitz (1974) for an analogous approach using differentiability.

${ }^{2}$ Note that the majority of the literature on risk aversion has been set in the context of the static portfolio problem, which is equivalent to the price of the risky good being random.
} 
We show that in the random income case as well as the case of the random price of the sure good, the effect of increasing risk aversion is to decrease the amount of the normal sure good. While if the price of the risky good is random, increasing risk aversion has an ambiguous effect on the amount of the sure good. These results follow from the fact that a more risk-averse individual is not concerned by the riskiness of the risky good, but rather by the riskiness of the utility gamble associated with the consumption bundle of the sure and risky goods. This is merely an implication of the expected utility maximization problem faced by the consumer. In fact, when the optimal amount of the risky good increases, it decreases the riskiness of the utility gamble faced by the individual. In other words, a concave transformation of the utility function implies that the more risk-averse individual prefers gambles whose corresponding utility gambles are less risky. This result pinpoints the rationale of the consumer's decisions not as a choice on the amount of risky versus sure goods, but rather as a choice on a set of utility gambles ordered in terms of their riskiness. ${ }^{3}$

Because the riskiness of the utility gambles yields the incentive for the consumer to choose an optimal gamble, through the maximization of expected utility, the counterintuitive result originally observed by Ross (1981) is achieved. Indeed, Ross (1981) provides an example showing that the ArrowPratt definition of risk aversion fails to deliver the right "intuitive" results. In particular, Ross (1981) writes that "in the portfolio problem, as wealth rises individuals whose risk aversion declines in the Arrow-Pratt sense do not necessarily increase their holding of riskier assets." Convinced of the intuitive idea that more risk aversion implies a smaller amount of the riskier asset (or good), Ross (1981) introduces a stronger measure of risk aversion that is necessary to accommodate the phenomenon observed when there are several independent sources of risk, i.e., that the more risk-averse individual actually chooses to consume more of the risky asset. In fact, in the example provided by Ross (1981), the individual chooses more of the risky asset (or good) because it reduces the riskiness of the utility gamble.

\footnotetext{
${ }^{3}$ Diamond and Stiglitz (1974) points out the relationship between increasing risk aversion and preferences ordering over utility gambles.
} 
In our general approach to the consumer problem, we relate classical demand theory to the theory of risk aversion. In particular, we show that the influence of risk aversion can be separated into independent components, i.e., income and substitution effects, as in classical demand theory with the outcome depending on their interplay. The interaction between the income and substitution effects as a determinant of the effect of risk aversion on optimal choice has also been noted in KM in the context of a consumersaving problem. KM shows that the effect of changing risk aversion depends on the rate of return on savings in the classical (certainty) case, that is, the impact of the interplay between the income and substitution effects. We generalize the KM result by showing that in a two-dimensional setting the implication of changing risk aversion depends on the source of randomness as well as the influence of ordinal effects, i.e., the income and substitution effects.

Our results on the effect of changes in risk aversion are summarized in three Propositions, which are inserted between relevant examples and figures that illustrate the Propositions. Proposition 1 studies the case in which income is random. Here, the implication of changes in risk aversion depends on the normality of the sure good. When the sure good is normal, the optimal decision of a more risk-averse individual is to consume more of the risky good, which decreases the riskiness of the associated utility gamble. It is precisely this case that contradicts the Ross intuition that a more risk-averse individual prefers more of the sure (or riskless) good. In Proposition 2, we show that, if the price of the normal sure good is random, a more risk-averse individual chooses more of the risky good, since the income and substitution effects pull in the same direction. Here, the pure substitution effect provides an incentive to consume less of the sure good. In this case, the utility gambles become less risky with more of the risky good, yielding an incentive for the consumer to move in that direction. Proposition 3 shows that, if the price of the normal risky good is random, as in the traditional portfolio problem, then the choice of the normal sure good is ambiguous. In this case, there is an incentive through the pure substitution effect to increase the amount of the sure good, i.e., increasing the sure good leads to a less risky utility gamble. Hence, the 
income and substitution effects pull in opposite directions, and depending on the relative strengths of these effects, the individual is led to consume more or less of the (normal) sure good. Note that Proposition 3 encompasses the Arrow-Pratt result, namely the risky good decreases with an increase in risk aversion, which is a special case and is due to the fact that, in the onedimensional case (i.e., perfect substitutes), there is no income effect, which implies that the utility gamble becomes less risky as the amount of the sure good is increased. However, when there is an income effect, this result holds only when the substitution effect is stronger than the income effect. ${ }^{4}$

Finally, note that there is a related literature that studies the effect of riskiness (using second-degree stochastic dominance) on optimal behavior originating with Diamond and Stiglitz (1974) and continuing with Kraus (1979) and Katz (1981). These papers study the effect of risk aversion on optimal behavior as an intermediate step in studying the effect of riskiness on optimal behavior. Although generic in nature, the results of the effect of risk aversion are important in their own right and are used in this paper to prove our results. Specifically, we use the results stated in Kraus (1979) and Katz (1981) to determine the effect of risk aversion for the consumer's problem. ${ }^{5}$ While the effect of risk aversion and the effect of riskiness are distinct issues, it is interesting to note that our results imply that for the consumer's problem, the income and substitution effects also have an influence on the comparative analysis for riskiness.

\footnotetext{
${ }^{4}$ In the three cases of randomness, an increase in risk aversion changes the weights attached to the marginal utilities corresponding to different outcomes of the uncertainty, while the income and substitution effects order the marginal utilities. For instance, with a normal good and uncertainty in income, the marginal utility is increasing in income, so that a lower income means a lower marginal utility and thus an increase in risk aversion adds more weight to the marginal utilities associated with lower incomes.

${ }^{5}$ In particular, we use Lemma 2 in Kraus (1979) which is derived from Theorem 4 in Diamond and Stiglitz (1974) showing the effect of risk aversion on optimal behavior. This lemma is used by Kraus (1979) as an intermediate step in determining the effect of riskiness of optimal behavior.
} 


\section{The Effect of Risk Aversion}

In this section, we study the effect of risk aversion on the optimal choice of the consumption profile $(x, \tilde{y}) \in \mathbb{R}_{+}^{2}$ with utility function $U(x, \tilde{y}), U_{1}, U_{2}>$ $0, U_{11}, U_{22}<0$. In the stochastic environment, $x$ is the sure good, while $\tilde{y}$ is the risky good due to the presence of randomness in the budget constraint. Using the KM utility representation, the consumer's maximization problem under uncertainty is

$$
\max _{x} W_{K M}(x, \tilde{y}(x))=\max _{x} \mathbb{E}_{\tilde{y}(x)} v_{K M}(U(x, \tilde{y}(x)))
$$

where $\mathbb{E}_{\tilde{y}(x)}$ is the expectation operator over $\tilde{y}(x)$, and $v_{K M}$ is a strictly increasing and concave function, $v_{K M}^{\prime}>0, v_{K M}^{\prime \prime} \leq 0$. Note that the risky good depends on $x$ through the budget constraint, i.e., $y(x)=\left(I-P_{x} x\right) / P_{y}$, where $I$ is income, and $P_{x}$ and $P_{y}$ are the prices of goods $x$ and $y$, respectively. The effect of risk aversion is studied in three different cases: random income, random price for the sure good, and random price for the risky good.

\subsection{Random Income}

When income is random, (1) is rewritten as

$$
\max _{x} \mathbb{E}_{\tilde{I}} v_{K M}\left(U\left(x, \frac{\tilde{I}-P_{x} x}{P_{y}}\right)\right)
$$

where $\mathbb{E}_{\tilde{I}}$ is the expectation operator for $\tilde{I}$. Proposition 1 states that the effect of risk aversion depends on the income effect when only income is random. The change in consumption due to a change in risk aversion does not result from a change in income as in the usual income effect. Instead Proposition 1

deals with the distribution of utilities associated with random income and the effect of that distribution of utilities on the choice of the consumption bundle as the consumer becomes more risk-averse. In particular, when the sure good is normal, a more risk-averse individual always consumes more of the risky good. 
Proposition 1. Given (2), a more risk-averse individual

1. decreases the amount of a normal good $x$,

2. increases the amount of an inferior good $x$, and

3. does not change the amount of good $x$ if there is no income effect.

Proof. See the appendix.

This counter-intuitive result is explained by the fact that the individual faces a utility gamble with each possible choice of the good $x$. The riskiness of the utility gamble is implicit in the optimal trade-off between the sure good and the risky good and is crucial to the choice of the individual, overshadowing the relevance of the riskiness of the good $\tilde{y}$. In fact, a more risk-averse individual chooses a level of consumption that reduces the riskiness of the utility levels associated with random income. To see this, we proceed in two steps. We first establish a relationship between the income effect and the types of utility gambles an individual faces. We then explain how optimal behavior is changed when risk-aversion increases. To that end, it is convenient to adopt a simple distribution for income, i.e., $\tilde{I} \sim(\pi \circ \underline{I},(1-\pi) \circ \bar{I})$, $\pi \in[0,1]$.

Income Effect and Utility Gambles. The income effect is key in explaining how changes in $x$ affect the riskiness of the utility gambles. To see this, let $x_{\underline{I}}$ and $x_{\bar{I}}$ be the optimal consumption for the sure good when $\pi=1$ and $\pi=0$, respectively. For nondegenerate distributions of income, $x \in\left[\min \left\{x_{\underline{I}}, x_{\bar{I}}\right\}, \max \left\{x_{\underline{I}}, x_{\bar{I}}\right\}\right]$ is the range of possible choices. We consider two cases.

Suppose that the sure good is normal, i.e., $x_{\underline{I}}<x_{\bar{I}}$, and let $M U(x, I) \equiv$ $U_{1}\left(x, \frac{I-P_{x} x}{P_{y}}\right)-U_{2}\left(x, \frac{I-P_{x} x}{P_{y}}\right) \frac{P_{x}}{P_{y}}$ be the marginal utility of consumption under income $I \in\{\underline{I}, \bar{I}\}$. Then, for any choice of $x$, the marginal utility under low income at the corresponding point of the lower budget constraint is smaller than the marginal utility under high income at the corresponding point of the upper budget constraint. Moreover, when the marginal utility under low income is tangent to the corresponding budget constraint, (i.e., $x=x_{\underline{I}}$ ), 


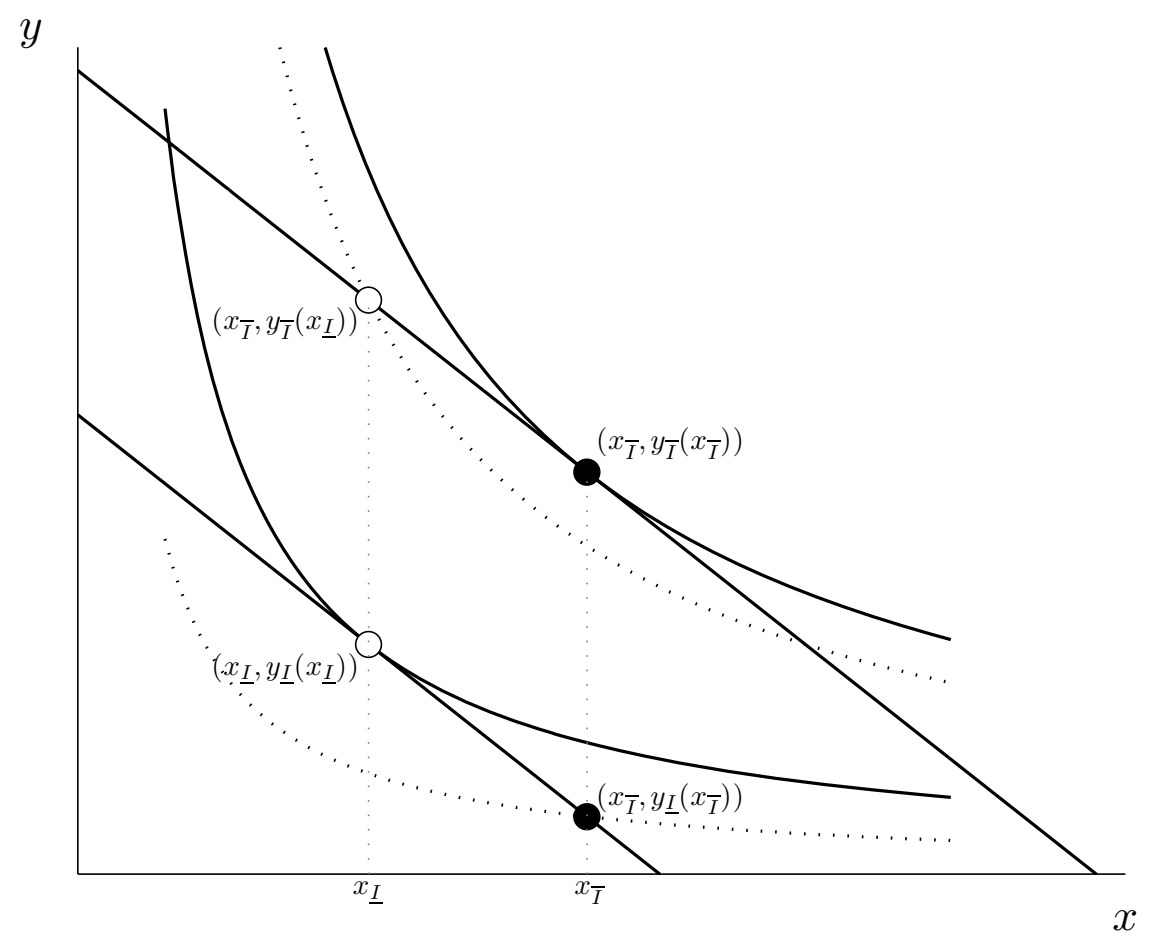

Figure 1: Utility Gambles with Normal Good $x$

then the marginal utility under high income is strictly positive. Hence, for $x \in\left[x_{\underline{I}}, x_{\bar{I}}\right]$, the difference between utility levels $U\left(x, \frac{\bar{I}-P_{x} x}{P_{y}}\right)-U\left(x, \frac{\underline{I}-P_{x} x}{P_{y}}\right)$ is positive and strictly increasing in $x \in\left[x_{\underline{I}}, x_{\bar{I}}\right]$. In other words, a decrease in $x$ brings the two utility levels closer together. In terms of gambles, this means that a decrease in $x$ results in a less risky utility gamble.

The relationship between $x$ and the riskiness of the utility gamble is shown in Figure 1 when the sure good is normal, i.e., $x_{\underline{I}}<x_{\bar{I}}$. The straight lines represent the budget constraints under low income and high income, while the convex lines are indifference curves. Note that the bundles $\left(x_{\underline{I}}, y_{\underline{I}}\left(x_{\underline{I}}\right)\right)$ and $\left(x_{\bar{I}}, y_{\bar{I}}\left(x_{\bar{I}}\right)\right)$ are the optimal bundles under certain low income and certain high income, respectively. ${ }^{6}$ When income is random, choosing $x$ implies

${ }^{6}$ For $I, I^{\prime} \in\{\underline{I}, \bar{I}\}$, let $y_{I}\left(x_{I^{\prime}}\right) \equiv\left(I-P_{x} x_{I^{\prime}}\right) / P_{y}$. 
choosing the utility gamble

$$
g(x) \equiv\left(\pi \circ U\left(x, \frac{I-P_{x} x}{P_{y}}\right),(1-\pi) \circ U\left(x, \frac{\bar{I}-P_{x} x}{P_{y}}\right)\right)
$$

for $x \in\left[x_{\underline{I}}, x_{\bar{I}}\right]$. From Figure 1 , the choice $x_{\bar{I}}$ has a utility gamble corresponding to the solid circles, while the choice $x_{\underline{I}}$ has a utility gamble corresponding to the empty circles. Hence, the gamble $g\left(x_{\underline{I}}\right)$ is less risky than the gamble $g\left(x_{\bar{I}}\right)$. In general, as shown in Figure 1 this implies that, for $x, x^{\prime} \in\left[x_{\underline{I}}, x_{\bar{I}}\right], x<x^{\prime}$,

$U\left(x^{\prime}, \frac{\underline{I}-P_{x} x^{\prime}}{P_{y}}\right)<U\left(x, \frac{\underline{I}-P_{x} x}{P_{y}}\right)<U\left(x, \frac{\bar{I}-P_{x} x}{P_{y}}\right)<U\left(x^{\prime}, \frac{\bar{I}-P_{x} x^{\prime}}{P_{y}}\right)$.

Suppose next that the sure good is inferior, i.e., $x_{\underline{I}}>x_{\bar{I}}$, so that the marginal utility under high income is smaller than the marginal utility under low income at the corresponding point on the budget constraint. For $x \in$ $\left[x_{\underline{I}}, x_{\bar{I}}\right]$, the difference between utility levels $U\left(x, \frac{\underline{I}-P_{x} x}{P_{y}}\right)-U\left(x, \frac{\bar{I}-P_{x} x}{P_{y}}\right)$ is positive and strictly decreasing in $x \in\left[x_{\underline{I}}, x_{\bar{I}}\right]$. In other words, an increase in $x$ brings the two utility levels closer together. In terms of gambles, this means that an increase in $x$ results in a less risky utility gamble, as depicted in Figure 2, where the utility gamble associated with $x^{*}$ is less risky than the utility gamble corresponding to $x$. In general, this implies that, for $x, x^{\prime} \in\left[x_{\bar{I}}, x_{\underline{I}}\right], x<x^{\prime}$,

$U\left(x, \frac{I-P_{x} x}{P_{y}}\right)<U\left(x^{\prime}, \frac{I-P_{x} x^{\prime}}{P_{y}}\right)<U\left(x^{\prime}, \frac{\bar{I}-P_{x} x^{\prime}}{P_{y}}\right)<U\left(x, \frac{\bar{I}-P_{x} x}{P_{y}}\right)$.

Optimal Utility Gamble. Having shown that the income effect determines the direction of a reduction in the riskiness of a gamble, we next turn to the optimal behavior. Without loss of generality, we define two different KM utility representations, $W_{K M}^{1}(x, \tilde{y}(x))=U(x, \tilde{y}(x))$ and $W_{K M}^{2}(x, \tilde{y}(x))=$ $\varphi(U(x, \tilde{y}(x))), \varphi^{\prime}>0, \varphi^{\prime \prime}<0$, so that $W_{K M}^{2}$ is strictly more risk-averse than $W_{K M}^{1}$.

Recall that $M U(x, I) \equiv U_{1}\left(x, \frac{I-P_{x} x}{P_{y}}\right)-U_{2}\left(x, \frac{I-P_{x} x}{P_{y}}\right) \frac{P_{x}}{P_{y}}$ is the marginal 


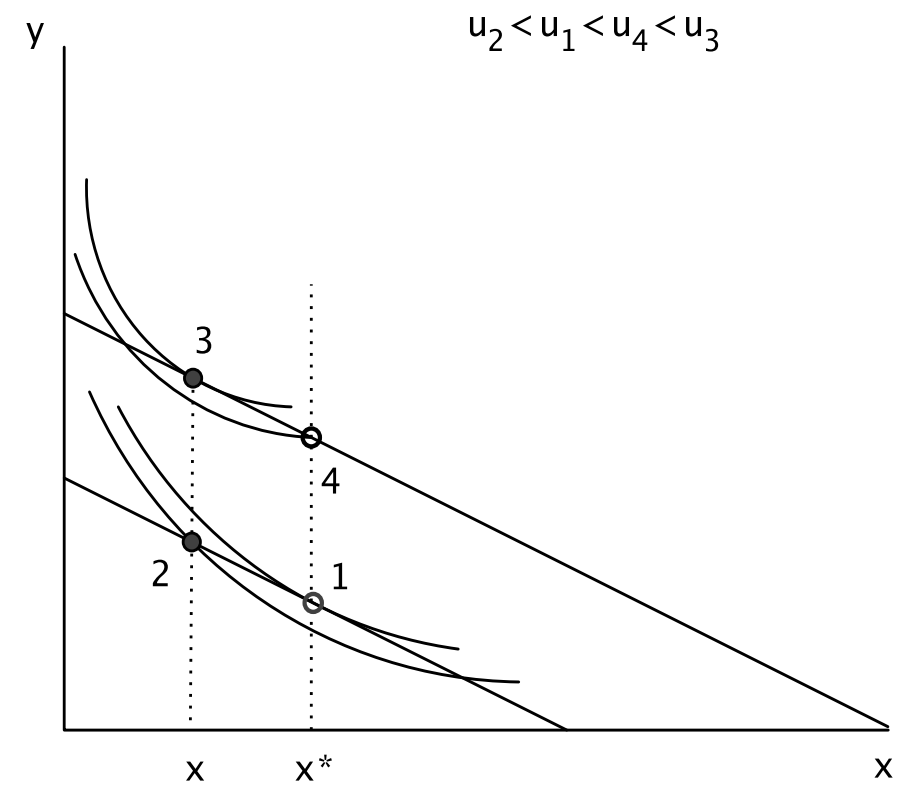

Figure 2: Utility Gambles with Inferior Good $x$ 
utility of consumption for $I \in\{\underline{I}, \bar{I}\}$. Then, the first-order conditions corresponding to preferences $W_{K M}^{1}$ and $W_{K M}^{2}$ are

$$
\pi M U(x, \underline{I})+(1-\pi) M U(x, \bar{I})=0,
$$

and

$$
\pi \rho\left(x, \underline{I}, \varphi^{\prime}\right) M U(x, \underline{I})+(1-\pi) \rho\left(x, \bar{I}, \varphi^{\prime}\right) M U(x, \bar{I})=0,
$$

respectively. Here,

$$
\rho\left(x, \underline{I}, \varphi^{\prime}\right)=\frac{\varphi^{\prime}\left(U\left(x, \frac{\underline{\underline{I}-P_{x} x}}{P_{y}}\right)\right)}{\varphi^{\prime}\left(U\left(x, \frac{\underline{I}-P_{x} x}{P_{y}}\right)\right)+\varphi^{\prime}\left(U\left(x, \frac{\bar{I}-P_{x} x}{P_{y}}\right)\right)},
$$

is a weighting function that depends on the risk aversion of the individual, $\rho\left(x, \underline{I}, \varphi^{\prime}\right)=1-\rho\left(x, \bar{I}, \varphi^{\prime}\right) \in[0,1]$. Note that risk-aversion measured by the function $\varphi$ enters the first-order condition only through the weighting function $\rho$. Remark 1 states the effect of risk aversion on the weighting function.

Remark 1. When income is random, the more risk-averse individual adds more weight to the low value of income, i.e., $\rho\left(x, \underline{I}, \varphi^{\prime}\right)>1 / 2$.

Given Remark 1, the effect of risk aversion is determined by the income effect, which orders the marginal utilities.

Remark 2. When the sure good is normal, $M U(x, \underline{I})<M U(x, \bar{I})$, while an inferior sure good yields $M U(x, \underline{I})>M U(x, \bar{I})$.

Combining Remarks 1 and 2 implies that a more risk-averse individual puts more weight on the lower marginal utility, which corresponds to the low income when the sure good is normal and the high income when the sure good is inferior. ${ }^{7}$ Hence, a more risk-averse agent decreases the amount of the sure good if and only if it is normal.

It is worth noting that before imposing the more risk-averse transformation $\varphi$, expected utility maximization yields a trade-off between the sure good

\footnotetext{
${ }^{7}$ This is a simplified version of the general proof of Kraus-Katz as used in the appendix.
} 


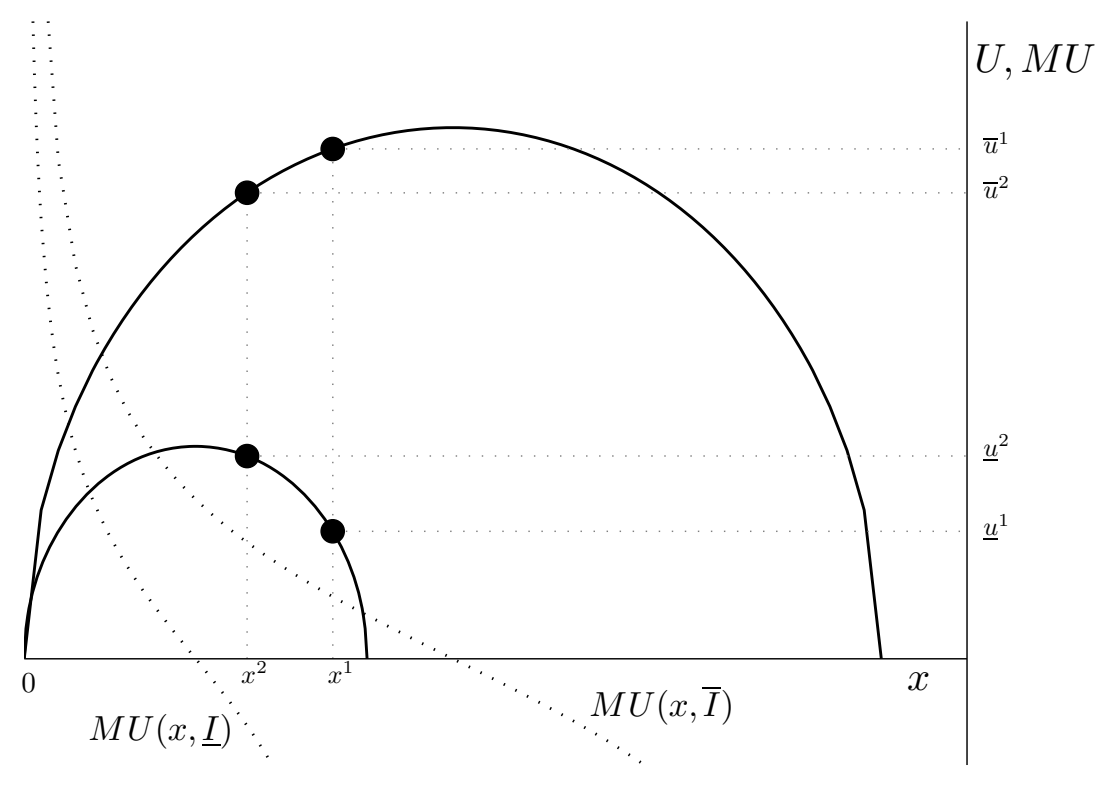

Figure 3: Optimal Utility Gamble with Normal Good $x$

and the risky good. However, the introduction of $\varphi$ changes that trade-off by giving the more risk-averse individual an incentive to choose a less risky utility gamble. In the random income case, this is done by reducing the amount of the good $x$. From this vantage point, it appears that the cardinality of the utility function determines the consumer's choice. However, it is clear from Figure 1, that, for any normal good $x$, it is ordinal preferences that dictate a decrease in the amount of the good $x$, which results in a less risky utility gamble.

Figure 3 further illustrates the effect of risk aversion on optimal behavior when preferences are Cobb-Douglas, i.e., $U(x, y)=x^{\alpha} y^{1-\alpha}, \alpha \in(0,1){ }^{8}$ Here, the sure good $x$ is normal. The solid lines represent the utility functions, while the dotted decreasing lines represent the marginal utility functions. The points $x^{1}$ and $x^{2}$ are the optimal bundles corresponding to preferences $W_{K M}^{1}$ and $W_{K M}^{2}$, respectively. From Figure 3, an increase in risk-aversion

\footnotetext{
${ }^{8}$ To generate the graph, we set $P_{x}=P_{y}=1$ and $I \in\{2,5\}$.
} 
adds more weight to the marginal utility under low income, which decreases the amount of the sure good, i.e., $x^{1}>x^{2}$, so as to reduce the riskiness of the utility gamble, i.e., $\underline{u}^{1}<\underline{u}^{2}<\bar{u}^{2}<\bar{u}^{1}$.

\subsection{Random Prices}

Having shown that the effect of risk aversion depends on the income effect when income is random, we next study the cases of random prices. Here, the relative strength of the income and substitution effects determine the effect of risk aversion on the optimal choice of $x$.

Random Price of the Sure Good. When the price of the sure good is random, (1) is rewritten as

$$
\max _{x} \mathbb{E}_{\tilde{P}_{x}} v_{K M}\left(U\left(x, \frac{I-\tilde{P}_{x} x}{P_{y}}\right)\right),
$$

where $\mathbb{E}_{\tilde{P}_{x}}$ is the expectation operator for $\tilde{P}_{x}$. Proposition 2 states that the effect of risk aversion is determined by the interplay of the income and substitution effects.

Proposition 2. Given (9), a more risk-averse individual

1. decreases the amount of a normal good $x$, and

2. increases the amount of an inferior good $x$ if and only if the income effect is stronger than the substitution effect.

Proof. See the appendix.

To explain the results in Proposition 2, it is convenient to adopt a simple distribution for the price of the sure good, i.e., $\tilde{P}_{x} \sim\left(\pi \circ \underline{P}_{x},(1-\pi) \circ \bar{P}_{x}\right)$, $\pi \in[0,1]$. Without loss of generality, we define two different KM utility representations, $W_{K M}^{1}(x, \tilde{y}(x))=U(x, \tilde{y}(x))$ and $W_{K M}^{2}(x, \tilde{y}(x))=\varphi(U(x, \tilde{y}(x)))$, $\varphi^{\prime}>0, \varphi^{\prime \prime}<0$, so that $W_{K M}^{2}$ is strictly more risk-averse than $W_{K M}^{1}$. 
Letting $M U\left(x, P_{x}\right) \equiv U_{1}\left(x, \frac{I-P_{x} x}{P_{y}}\right)-U_{2}\left(x, \frac{I-P_{x} x}{P_{y}}\right) \frac{P_{x}}{P_{y}}$ be the marginal utility of consumption for $P_{x} \in\left\{\underline{P}_{x}, \bar{P}_{x}\right\}$, the first-order conditions corresponding to preferences $W_{K M}^{1}$ and $W_{K M}^{2}$ are

$$
\pi M U\left(x, \underline{P}_{x}\right)+(1-\pi) M U\left(x, \bar{P}_{x}\right)=0
$$

and

$$
\pi \rho\left(x, \underline{P}_{x}, \varphi^{\prime}\right) M U\left(x, \underline{P}_{x}\right)+(1-\pi) \rho\left(x, \bar{P}_{x}, \varphi^{\prime}\right) M U\left(x, \bar{P}_{x}\right)=0,
$$

respectively. Here,

$$
\rho\left(x, \underline{P}_{x}, \varphi^{\prime}\right)=\frac{\varphi^{\prime}\left(U\left(x, \frac{I-\underline{P}_{x} x}{P_{y}}\right)\right)}{\varphi^{\prime}\left(U\left(x, \frac{I-\underline{P}_{x} x}{P_{y}}\right)\right)+\varphi^{\prime}\left(U\left(x, \frac{I-\bar{P}_{x} x}{P_{y}}\right)\right)},
$$

is a weighting function that depends on the risk aversion of the individual, $\rho\left(x, \underline{P}_{x}, \varphi^{\prime}\right)=1-\rho\left(x, \bar{P}_{x}, \varphi^{\prime}\right) \in[0,1]$. Note that risk-aversion measured by the function $\varphi$ enters the first-order condition only through the weighting function $\rho$, as in the case of random income. Remark 3 states the effect of risk-aversion on the weighting function when $P_{x}$ is random.

Remark 3. When the price of the sure good is random, the more risk-averse individual adds less weight to the low value of $P_{x}$, i.e., $\rho\left(x, \underline{P}_{x}, \varphi^{\prime}\right)<1 / 2$.

The effect of risk aversion is determined by the income and substitution effects, which orders the marginal utilities. Consider the sign of the derivative of $M U\left(x, P_{x}\right)$ with respect to $P_{x}$ is useful in ordering the marginal utilities, i.e.,

$$
\frac{\partial M U\left(x, P_{x}\right)}{\partial P_{x}}=\underbrace{-\left(U_{12}-U_{22} \frac{P_{x}}{P_{y}}\right) \frac{x}{P_{y}}}_{=I E_{P_{x}}} \underbrace{-\frac{U_{2}}{P_{y}}}_{=S E_{P_{x}}} .
$$

Here, $I E_{P_{x}}$ and $S E_{P_{x}}$, both negative, are proportional to and of the same sign as the income effect and the substitution effect, respectively.

Remark 4. When the sure good is normal, both the income and substitution effects are negative, so that $M U\left(x, \underline{P}_{x}\right)>M U\left(x, \bar{P}_{x}\right)$. When the sure good is 


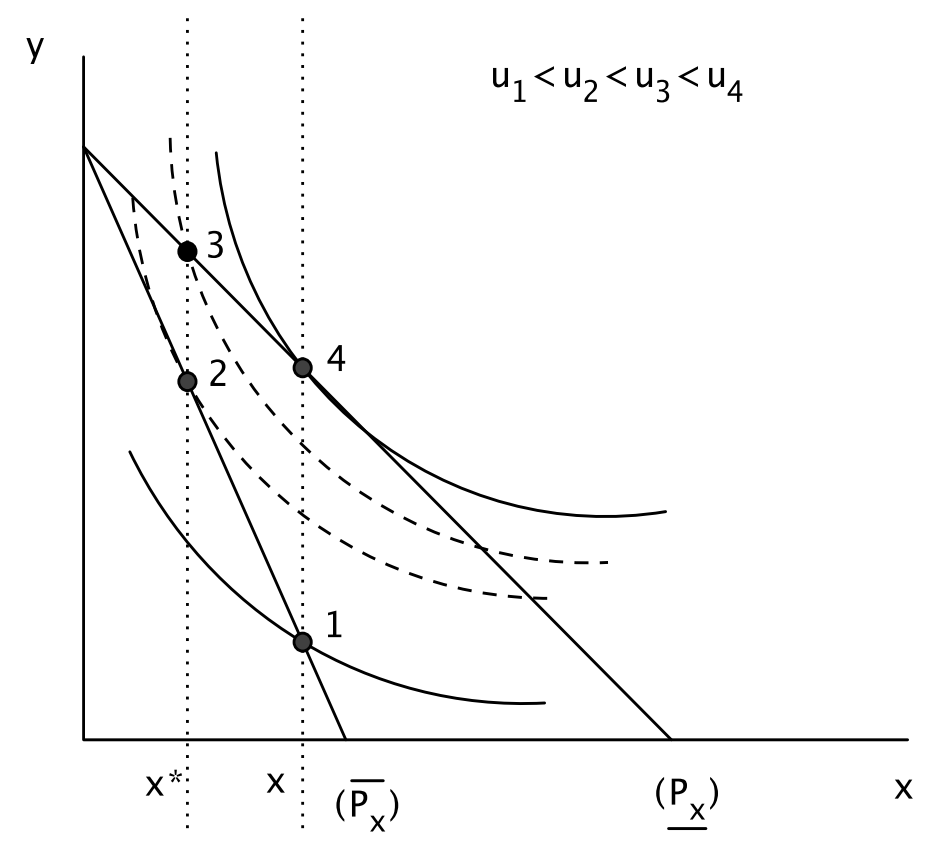

Figure 4: Utility Gambles with Normal Good $x$ and Random $P_{x}$

inferior, i.e., $I E_{P_{x}}>0$, the relative strengths of the income and substitution effects determine the ordering of the marginal utilities. For instance, if the (positive) income effect is stronger than the (negative) substitution effect, then, from (13), $M U\left(x, \underline{P}_{x}\right)<M U\left(x, \bar{P}_{x}\right)$.

Remarks 3 and 4 explain the result in Proposition 2. In particular, when the sure good is normal, a more risk-averse individual puts less weight on the marginal utility corresponding to the high price of $x$. Proposition 2 is illustrated in Figure 4 for the case of a normal good $x$. Due to the randomness of $P_{x}$, the slope of the budget constraint makes the utility gamble less risky as consumption decreases. Specifically, when $P_{x}$ is random, the pure substitution effect induces a less risky utility gamble in the direction of less quantity of the sure good, from $x$ to $x^{*}$, which increases the amount of the risky good $y$. 


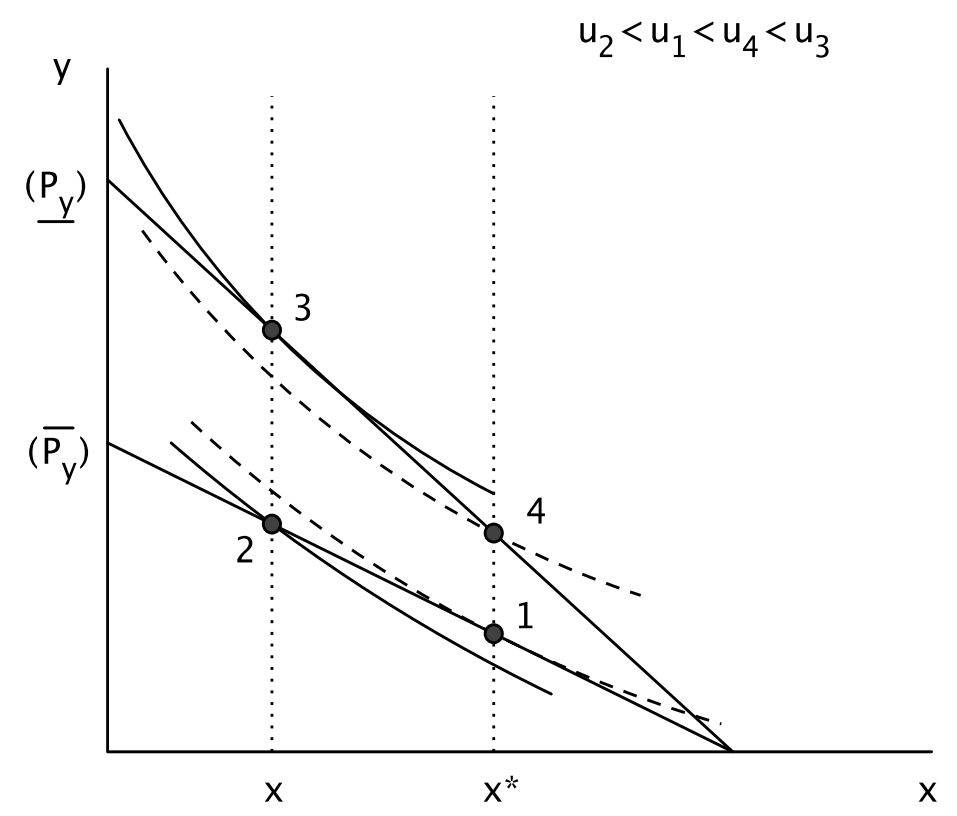

Figure 5: Utility Gambles with Normal Good $x$ and Random $P_{y}$

Random Price of the Risky Good. When the price of the risky good is random, (1) is rewritten as

$$
\max _{x} \mathbb{E}_{\tilde{P}_{y}} v_{K M}\left(U\left(x, \frac{I-P_{x} x}{\tilde{P}_{\tilde{y}}}\right)\right),
$$

where $\mathbb{E}_{\tilde{P}_{y}}$ is the expectation operator for $\tilde{P}_{y}$. Proposition 3 states that the effect of risk aversion is again determined by the interplay of the income and substitution effects.

Proposition 3. Given (14), a more risk-averse individual

1. decreases the amount of a normal good $x$ if and only if the income effect is stronger than the substitution effect, and

2. increases the amount of an inferior good $x$. 
Proof. See the appendix.

Proposition 3 is illustrated in Figure 5, which shows that, when the price of the risky good is random, the substitution effect induces a less risky utility gamble by increasing the amount of the sure good from $x$ to $x^{*}$.

\subsection{Examples}

Propositions 1, 2, and 3 establish the connection between risk aversion and classical demand theory implicit in KM, and explain, in that context, the limits of the intuition of the Ross (1981) critique on risk aversion. In particular, increasing the amount of the risky good when risk aversion increases, is natural and not counterintuitive, as thought by Ross (1981) and his followers. We now illustrate our results by considering specific classes of preferences: Cobb-Douglas, Leontief, and quasi-linear utility functions.

Suppose that preferences are Cobb-Douglas, i.e., $U(x, y)=x^{\alpha} y^{\beta}, \alpha, \beta>$ 0 , so that $x$ is a normal good. Note that the results in Propositions 1,2 , and 3 continue to hold, even when the utility function is concave or convex, as long as there is an interior solution to the constrained optimization problem. For random income, more risk aversion has the effect of decreasing the amount of $x$. This result is even stronger when the price of $x$ is random, since the income and the substitution effects go in the same direction. However, when the price of $y$ is random, the income and the substitution effects not only go in opposite directions, but cancel each other out with Cobb-Douglas preferences. This is exactly the consumption-saving problem discussed in $\mathrm{KM}$, in which the rate of return (the price of $y$ ) is random.

Suppose next that preferences are Leontief, i.e., $U(x, y)=u(\min \{x, y\})$, $u^{\prime}>0, u^{\prime \prime}<0$, so that $x$ is a normal good. Then, there is no substitution effect and the income effect determines the direction of the change along with an increase in risk aversion. In particular, regardless of the source of risk, an increase in risk aversion always decreases the amount of the sure good in favor of the risky good. To see this, consider the income distribution $\tilde{I} \sim(1-\pi \circ \underline{I}, \pi \circ \bar{I}), \underline{I}<\bar{I}$. Moreover, assume that $P_{x}=P_{y}=1$. Since the optimal solution lies in $\underline{I} / 2 \leq x \leq \bar{I} / 2$, the individual's maximization 
problem is ${ }^{9}$

$$
\max _{x} \mathbb{E}_{\tilde{I}} u(\min \{x, \tilde{I}-x\})=\pi u(x)+(1-\pi) u(\underline{I}-x) .
$$

The first-order condition is $\pi u^{\prime}(x)-(1-\pi) u^{\prime}(\underline{I}-x)=0$ so that

$$
\frac{u^{\prime}(x)}{u^{\prime}(\underline{I}-x)}=\frac{1-\pi}{\pi}
$$

Consider next a more risk-averse individual, i.e., the more risk-averse individual's maximization problem is

$$
\max _{x} \pi \varphi(u(x))+(1-\pi) \varphi(u(\underline{I}-x)),
$$

$\varphi^{\prime}>0, \varphi^{\prime \prime}<0$. The first-order condition is $\pi \varphi^{\prime}\left(u\left(x^{*}\right)\right) u^{\prime}\left(x^{*}\right)-(1-\pi) \varphi^{\prime}(u(\underline{I}-$ $\left.\left.x^{*}\right)\right) u^{\prime}\left(\underline{I}-x^{*}\right)=0$ so that

$$
\frac{u^{\prime}\left(x^{*}\right)}{u^{\prime}\left(\underline{I}-x^{*}\right)}=\frac{1-\pi}{\pi} \frac{\varphi^{\prime}\left(u\left(\underline{I}-x^{*}\right)\right)}{\varphi^{\prime}\left(u\left(x^{*}\right)\right)} .
$$

Since $\varphi$ is strictly concave and $\underline{I} / 2 \leq x^{*} \leq \bar{I} / 2$, it follows that

$$
\frac{\varphi^{\prime}\left(u\left(\underline{I}-x^{*}\right)\right)}{\varphi^{\prime}\left(u\left(x^{*}\right)\right)}>1 .
$$

Hence, from (16), (18), and (19),

$$
\frac{u^{\prime}\left(x^{*}\right)}{u^{\prime}\left(\underline{I}-x^{*}\right)}=\frac{1-\pi}{\pi} \frac{\varphi^{\prime}\left(u\left(\underline{I}-x^{*}\right)\right)}{\varphi^{\prime}\left(u\left(x^{*}\right)\right)}>\frac{u^{\prime}(x)}{u^{\prime}(\underline{I}-x)}
$$

which implies that $u^{\prime}\left(x^{*}\right)>u^{\prime}(x)$ or $x^{*}<x$ as shown in Figure 6 .

The more risk-averse individual consumes even more of the risky good

\footnotetext{
${ }^{9}$ If $x<\underline{I} / 2$, then the outcome is strictly worse than choosing $x=\underline{I} / 2$, while, if $x>\bar{I} / 2$, then the outcome is strictly worse than choosing $x=\bar{I} / 2$. In this case, the optimal solution has both $x$ and $y$ positive, i.e., there is no corner solution in which either $x=0$ or $y=0$. However, on the interval $\underline{I} / 2 \leq x \leq \bar{I} / 2$, there can be corner solutions, when $x=\underline{I} / 2$ or $x=\bar{I} / 2$, which correspond to the most risk-averse and the most risk-loving choices respectively.
} 


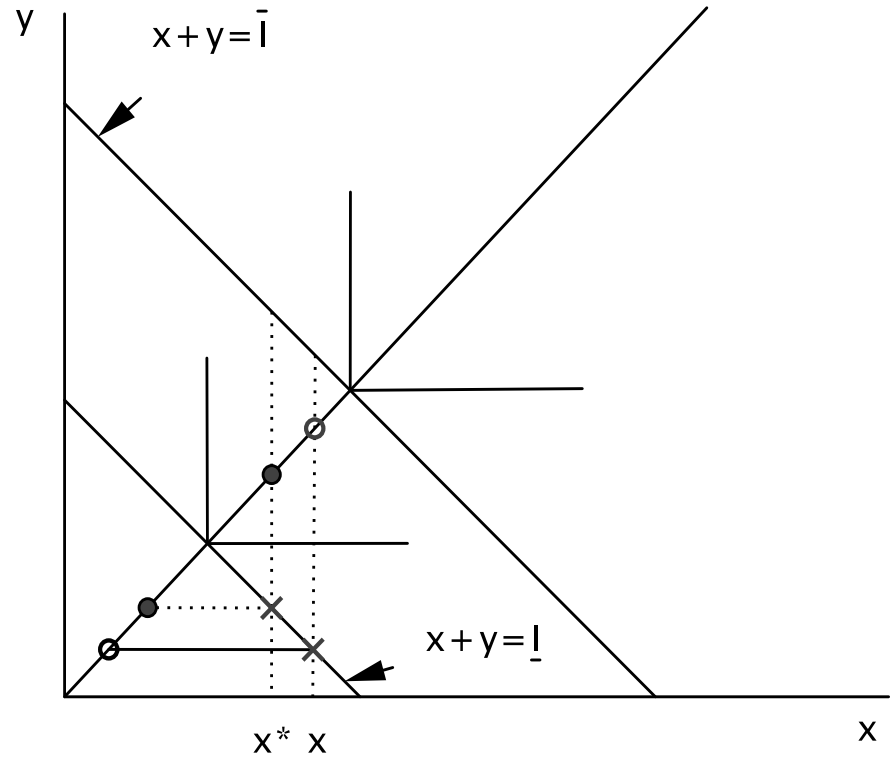

Figure 6: Leontief Preferences 
due to the presence of only income effects. ${ }^{10}$ This decrease in the amount of the sure good $x$ yields a less risky utility gamble as depicted in Figure 6. Note that, with Leontief preferences, the amount of $x$ always decreases because there is only a pure income effect, i.e., without substitution. That is why Leontief preferences yield results opposite to the Arrow-Pratt's portfolio problem, in which there is no income effect and only substitution effect.

Suppose finally that preferences are quasi-linear, where $x$ is a normal good. These preferences shed light on the Arrow-Pratt result, i.e., an increase in risk aversion increases the amount of income invested in the safe asset (i.e., allocated to the sure good in our context). We now demonstrate that the Arrow-Pratt result holds due to the absence of the income effect, and that the source of uncertainty lies in the rate of return of the risky asset. Specifically, we consider two cases and show stark difference in results between the two. First, consider the case in which there is no income effect for the sure good $x$, i.e., $U(x, y)=u_{1}(x)+y, u_{1}^{\prime}>0, u_{1}^{\prime \prime}<0$. When income is random, since there is no income effect, risk aversion has no effect on the amount of $x$. When the price of $x$ is random, increased risk aversion causes the amount of $x$ to decrease solely due to the substitution effect. However, for a random price of the risky good, the substitution effect dominates (since there is no income effect), which implies that the amount of $x$ increases along with an increase in risk aversion. This result generalizes the result in the Arrow-Pratt portfolio problem. In fact, it is only in this case that increasing risk aversion increases the amount of the sure good without reference to income and substitution effects. However, the result is not robust to a slight modification in the utility function. To see this, consider the quasi-linear utility function, i.e., $U(x, y)=x+u_{2}(y), u_{2}^{\prime}>0, u_{2}^{\prime \prime}<0$. In this case, the sure good $x$ is normal, so that if either income or the price of $x$ is random, risk aversion decreases the amount of the sure good $x$. On the other hand, for random price of the risky good, the income and substitution effects pull in opposite directions. If the income effect is dominant, then an increase in risk aversion leads to a

\footnotetext{
${ }^{10}$ Note that if the initial choice is $x=\underline{I} / 2$, then the consumer is making the most risk-averse choice. Therefore a more risk-averse transformation cannot reduce the level of $x$.
} 
decrease in the amount of the sure good $x$. This last result illustrates that the Arrow-Pratt result is solely due to the absence of an income effect on $x$. Finally, note that another version of the Arrow-Pratt theorem is that, if income increases, then an individual with decreasing risk aversion reduces the amount of the sure good. This result is not general and is due only to the fact that, in the portfolio problem, there is no income effect for the sure good.

\section{Final Remarks}

In this paper, we completely characterize the relationship between changes in risk aversion and classical demand theory in the case of a single source of uncertainty. We show that a more risk-averse consumer generally decreases the amount placed in the sure good. In addition, we show that it is the utility gambles that determine the choice of a more risk-averse agent between the sure good and the risky good. This provides an explanation for certain paradoxical behaviors of an individual who becomes more risk-averse. The paper also paves a path for some immediate interesting questions. In particular, one could ask what the relationship between risk aversion and classical demand theory implies for changes in income in which the consumer is decreasingly risk-averse. This is especially interesting in light of Arrow-Pratt's result that in the portfolio case increasing income results in an increase in the risky asset if and only if the consumer is decreasingly risk-averse with income. 


\section{A Proofs}

We combine the proofs of Propositions 1, 2, and 3. We first state the following Lemma which combines Lemma 2 in Kraus (1979) and conditions stated in Katz (1981).

Kraus-Katz Lemma. Let $x_{\tilde{\theta}}^{*}=\arg \max _{x} \mathbb{E}_{\tilde{\theta}} \varphi(Z(x, \tilde{\theta}))$ such that $\partial^{2} \varphi(Z(x, \theta)) /\left.\partial x^{2}\right|_{x=x_{\tilde{\theta}}^{*}}<0$. If $\partial Z(x, \theta) /\left.\partial \theta\right|_{x=x_{\tilde{\theta}}^{*}}>0$ and $\partial^{2} Z(x, \theta) /\left.\partial x \partial \theta\right|_{x=x_{\tilde{\theta}}^{*}}<$ $0(>0)$, then $x_{\tilde{\theta}}^{*}$ increases (decreases) along with an increase in risk aversion. Alternatively, if $\partial Z(x, \theta) /\left.\partial \theta\right|_{x=x_{\tilde{\theta}}^{*}}<0$ and $\partial^{2} Z(x, \theta) /\left.\partial x \partial \theta\right|_{x=x_{\tilde{\theta}}^{*}}>0(<0)$, then $x_{\tilde{\theta}}^{*}$ increases (decreases) along with an increase in risk aversion.

In the consumer's problem, $Z(x, \theta) \equiv U\left(x,\left(I-P_{x} x\right) / P_{y}\right), \theta \in\left\{I, P_{x}, P_{y}\right\}$. Suppose first that income is random as in Proposition 1, i.e., $\tilde{\theta} \equiv \tilde{I}$. Then, $\frac{\partial U\left(x,\left(I-P_{x} x\right) / P_{y}\right)}{\partial I}>0$, and $\frac{\partial^{2} U\left(x,\left(I-P_{x} x\right) / P_{y}\right)}{\partial x \partial I}=I E_{I} \equiv U_{12} \cdot \frac{x}{P_{y}}-U_{22} \cdot \frac{P_{x} x}{P_{y}^{2}}$ is of the same sign as the income effect related to a change in income. By Kraus-Katz Lemma, $x_{\tilde{I}}^{*}$ decreases along with an increase in risk aversion when the sure good is normal, i.e., $\left.I E_{I}\right|_{x=x_{\tilde{I}}^{*}}>0$. Suppose next that the price of the sure good is random as in Proposition 2, i.e., $\tilde{\theta} \equiv \tilde{P}_{x}$. Then, $\frac{\partial U\left(x,\left(I-P_{x} x\right) / P_{y}\right)}{\partial P_{x}}<0$, and $\frac{\partial^{2} U\left(x,\left(I-P_{x} x\right) / P_{y}\right)}{\partial x \partial P_{x}}=I E_{P_{x}}+S E_{P_{x}}$, where $I E_{P_{x}} \equiv-\left[U_{12} \cdot \frac{x}{P_{y}}-U_{22} \cdot \frac{P_{x} x}{P_{y}^{2}}\right]$ and $S E_{P_{x}} \equiv-\frac{U_{2}}{P_{y}}<0$ are of the same sign as the income and substitution effects, respectively, related to a change in the price of the sure good. By Kraus-Katz Lemma, $x_{\tilde{P}_{x}}^{*}$ decreases along with an increase in risk aversion when the sure good is normal, i.e., $\left.I E_{P_{x}}\right|_{x=x_{\tilde{P}_{x}}^{*}}<0$. The effect of risk aversion for an inferior sure good depends on the relative strength of the income and substitution effects. Suppose finally that the price of the risky good is random as in Proposition 3, i.e., $\tilde{\theta} \equiv \tilde{P}_{y}$. Then, $\frac{\partial U\left(x,\left(I-P_{x} x\right) / P_{y}\right)}{\partial P_{y}}<0$, and $\frac{\partial^{2} U\left(x,\left(I-P_{x} x\right) / P_{y}\right)}{\partial x \partial P_{y}}=I E_{P_{y}}+S E_{P_{y}}$ where $I E_{P_{y}} \equiv-\left[U_{12} \cdot \frac{x}{P_{y}}-U_{22} \cdot \frac{P_{x}}{P_{y}}\right] \frac{I-P_{x} x}{P_{y}^{2}}$ and $S E_{P_{y}} \equiv \frac{U_{2} P_{x}}{P_{y}^{2}}>0$ are of the same sign as the income and substitution effects, respectively, related to a change in the price of the risky good. By Kraus-Katz Lemma, $x_{\tilde{P}_{y}}^{*}$ increases along with an increase in risk aversion when the good is inferior, i.e., $\left.I E_{P_{y}}\right|_{x=x_{\tilde{P}_{y}}^{*}}<0$. The effect of risk aversion for a normal sure good depends on the relative strength of the income and substitution effects. 


\section{References}

K.J. Arrow. Aspects of the Theory of Risk-Bearing. Yrjo Jahnssonin Saatio, 1965.

P.A. Diamond and J.E. Stiglitz. Increases in Risk and in Risk Aversion. J. Econ. Theory, 8(3):337-360, 1974.

E. Katz. A Note on a Comparative Statics Theorem for Choice under Risk. J. Econ. Theory, 25(2):318-319, 1981.

R.E. Kihlstrom and L.J. Mirman. Risk Aversion with Many Commodities. J. Econ. Theory, 8(3):361-388, 1974.

M. Kraus. A Comparative Statics Theorem for Choice under Risk. J. Econ. Theory, 21(3):510-517, 1979.

J.W. Pratt. Risk Aversion in the Small and in the Large. Econometrica, 32 (1-2):122-136, 1964.

S.A. Ross. Some Stronger Measures of Risk Aversion in the Small and the Large with Applications. Econometrica, 49(3):621-638, 1981. 